\title{
Clonogens and cancer stem cells
}

\section{Michael Baumann, Mechthild Krause and Richard Hill}

We would like to thank Maurice Tubiana and Serge Koscielny for their comments on our Opinion article (Exploring the role of cancer stem cells in radioresistance. Nature Rev. Cancer 8, 545-554 (2008)) ${ }^{1}$, which raise some important issues (On clonogenic tumour cells and metastasis-forming cells Nature Rev. Cancer 8, 990 (2008)) $)^{2}$.

We have chosen not to use the terms colony-forming unit and clonogenic cell as synonyms for cancer stem cells, because these terms, in our opinon, may potentially be confused with cells characterized by their ability to form colonies of more than 50 daughter cells in culture. There is significant evidence that there are more cells that can form colonies in culture than would be regarded as stem cells based on in vivo testing (bone marrow is an excellent example, in addition to examples for tumour cells and xenografts $\left.{ }^{3,4}\right)$. However, as we point out in our article, culture conditions and transplant conditions can affect the numbers obtained in such studies. Furthermore, fetal calf serum is reported to induce differentiation $^{5,6}$, and it has been suggested that it may affect what is analysed in clonogenic assays in vitro. The studies mentioned by Tubiana and Koscielny are important and, with the limitations mentioned above, indeed provide further support for the hypothesis that cancer stem cells might have different biological behaviour and different radiosensitivity compared with non-stem cells. The first studies provide evidence that the cell-cycle time is different between clonogenic and non-clonogenic tumour cells in vitro $^{7-9}$. It would be interesting to explore whether similar differences exist between cancer stem cells and non-stem cells in vivo. To our knowledge, there are currently no data on this question.
The second comment by Tubiana and Koscielny adds a further interesting aspect to the discussion about the function of cancer stem cells in vivo. We fully agree that, by definition, clinical metastases are initiated by cancer stem cells. It is well-known that only a small fraction of cells released from tumours ever form metastases. It can be argued that this is because most of the cells released are not stem cells, but this is currently speculation. The fact that the probability of distant metastatic dissemination does not increase linearly with the size of the primary tumour ${ }^{10,11}$ is an important finding, as other data indicate that the number of cancer stem cells increases linearly with the tumour volume ${ }^{12-14}$. The data might be interpreted as indicating that the relative proportion of cancer stem cells decreases with increasing tumour volume. However, other influences might also be important. For example, the invasive front of the tumour, for geometrical reasons, is relatively larger in small tumours. Also, increasingly impaired supply with tumour blood vessels might lead to a reduction of the relative metastatic potential per stem cell with increasing tumour volume. In addition, it cannot be ruled out that the primary tumour may have been releasing anti-angiogenic factors, which prevented metastatic growth, as demonstrated by Folkman's studies on angiostatin and endostatin ${ }^{15,16}$. Presumably, the larger the primary tumour, the more such factors may be released.

Overall, the data gained by Tubiana and Koscielny are important for the discussion of cancer stem cells and provide further interesting starting points for future stem-cell-related research.

Michael Baumann and Mechthild Krause are at the Department of Radiation Oncology and OncoRay
Center for Radiation Research in Oncology, and Michael Baumann is also at the University Cancer Center, Medical Faculty and University Hospital Carl Gustav Carus, Technische Universität Dresden, Fetscherstrasse 74, 01307 Dresden, Germany.

Richard Hill is at the Department of Medical Biophysics, Princess Margaret Hospital, Ontario Cancer Institute, University of Toronto, 610 University Avenue, Toronto, Ontario M5G 2M9, Canada.

Correspondence to M.B. e-mail: michael.baumann@uniklinikum-dresden.de

1. Baumann, M., Krause, M. \& Hill, R. Exploring the role of cancer stem cells in radioresistance. Nature Rev. Cancer 8, 545-554 (2008).

2. Tubiana, M. \& Koscielny, S. On clonogenic tumour cells and metastasis-forming cells. Nature Rev. Cancer 8, 990 (2008).

3. Denekamp, J. Tumour stem cells: facts, interpretation and consequences. Radiother. Oncol. 30, 6-10 (1994). Pereira, C., Clarke, E. \& Damen, J. Hematopoietic colony-forming cell assays. Methods Mol. Biol. 407 177-208 (2007).

5. Yokoyama, M., Miwa, H., Maeda, S., Wakitani, S. \& Takagi, M. Influence of fetal calf serum on differentiation of mesenchymal stem cells to chondrocytes during expansion. J. Biosci. Bioeng. 106, 46-50 (2008).

6. Hisanaga, E. et al. A simple method to induce differentiation of murine bone marrow mesenchymal cells to insulin-producing cells using conophylline and betacellulin-delta4. Endocr. J. 55, 535-543 (2008).

7. Demeestere, M., Rockwell, S., Valleron, A. J., Frindel, E. \& Tubiana, M. Cell proliferation in EMT6 tumours treated with single doses of X-rays or hydroxyurea. II. Computer simulations. Cell Tissue Kinet. 13, 309-317 (1980).

8. Rockwell, S., Frindel, E. \& Tubiana, M. A technique for determining the proportion of the clonogenic cells in $\mathrm{S}$ phase in ENT6 cell cultures and tumors. Cell Tissue Kinet. 9, 313-323 (1976).

9. Rockwell, S., Frindel, E., Valleron, A. J. \& Tubiana, M. Cell proliferation in EMT6 tumors treated with single doses of $x$-rays or hydroxyurea. I. Experimental results. Cell Tissue Kinet. 11, 279-289 (1978).

10. Koscielny, S. \& Tubiana, M. The link between local recurrence and distant metastases in human breast cancer. Int. J. Radiat. Oncol. Biol. Phys. 43, 11-24 (1999).

11. Tubiana, M. \& Koscielny, S. Natural history of human breast cancer: recent data and clinical implications. Breast Cancer Res. Treat. 18, 125-140 (1991).

12. Baumann, M., Dubois, W. \& Suit, H. D. Response of human squamous cell carcinoma xenografts of different sizes to irradiation: relationship of clonogenic cells, cellular radiation sensitivity in vivo, and tumor rescuing units. Radiat. Res. 123, 325-330 (1990).

13. Kummermehr, J. \& Trott, K. R. in Stem Cells (ed. Potten, C. S.) 363-400 (Academic Press, London, 1997).

14. Suit, H., Shalek, R. \& Wette, R. in Cellular Radiation Biology 514-530 (Williams and Wilkins, Baltimore, 1965).

15. O'Reilly, M. S. et al. Angiostatin: a novel angiogenesis inhibitor that mediates the suppression of metastases by a Lewis lung carcinoma. Cell 79, 315-328 (1994).

16. O'Reilly, M. S. et al. Endostatin: an endogenous inhibitor of angiogenesis and tumor growth. Cell $\mathbf{8 8}$ 277-285 (1997) 